\title{
New bounds on the maximum number of points on genus- 4 curves over small finite fields
}

\author{
Everett W. Howe \\ Dedicated to the memory of my mother, Norma Howe
}

\begin{abstract}
For prime powers $q<100$, we compute new upper and lower bounds on $N_{q}(4)$, the maximal number of points on a genus- 4 curve over a finite field with $q$ elements. We determine the exact value of $N_{q}(4)$ for 17 prime powers $q$ for which the value was previously unknown.
\end{abstract}

\section{Introduction}

For every prime power $q$ and integer $g \geq 0$, let $N_{q}(g)$ denote the maximal number of points on a curve of genus $g$ over $\mathbf{F}_{q}$. In this paper we compute new upper and lower bounds on $N_{q}(4)$ for prime powers $q$ less than 100 , and we find the exact value of $N_{q}(4)$ for 17 prime powers $q$ for which the value was previously unknown.

Weil's famous 'Riemann Hypothesis' for curves over finite fields [22 25] gives the fundamental result that

$$
N_{q}(g) \leq q+1+2 g \sqrt{q},
$$

which generalizes Hasse's theorem for the number of points on an elliptic curve over a finite field [6. Weil's bound was improved significantly in the case where $g$ is large with respect to $q$ by Manin [14, Ihara 11, and Drinfel'd and Vlădut [20, and for fixed $q$ we have the Drinfel'd-Vlădut bound

$$
N_{q}(g) \leq(\sqrt{q}-1+o(1)) g \quad \text { as } g \rightarrow \infty .
$$

When $q$ is a square this bound is asymptotically optimal, in the sense that

$$
\lim \sup N_{q}(g) / g=\sqrt{q}-1 .
$$

The value of this lim sup is not known for any nonsquare $q$.

For any particular choice of $q$ and $g$, it can be a difficult computational problem to determine the actual value of $N_{q}(g)$ and to find a curve that attains this number of points. When $g$ is less than $(q-\sqrt{q}) / 2$, the best upper bound known is often Serre's improvement to the Weil bound [17:

$$
N_{q}(g) \leq q+1+g\lfloor 2 \sqrt{q}\rfloor .
$$

2010 Mathematics Subject Classification. Primary 11G20; Secondary 14G05, 14G10, 14G15.

Key words and phrases. Curve, rational point, zeta function, finite field. 


\begin{tabular}{|c|c|c|c|c|c|c|c|c|}
\hline$q$ & old & new & $q$ & old & new & $q$ & old & new \\
\hline 2 & 8 & 8 & 23 & -58 & 57 & 59 & -118 & 116 \\
\hline 3 & 12 & 12 & 25 & 66 & 66 & 61 & -122 & $118-119$ \\
\hline 4 & 15 & 15 & 27 & 64 & 64 & 64 & 129 & 129 \\
\hline 5 & 18 & 18 & 29 & -70 & $67-68$ & 67 & -132 & 129 \\
\hline 7 & 24 & 24 & 31 & -73 & 72 & 71 & $132-136$ & 134 \\
\hline 8 & 25 & 25 & 32 & $71-72$ & $71-72$ & 73 & -139 & 138 \\
\hline 9 & 30 & 30 & 37 & -84 & 82 & 79 & -148 & 148 \\
\hline 11 & $33-34$ & 33 & 41 & -90 & 88 & 81 & 154 & 154 \\
\hline 13 & -39 & 38 & 43 & -93 & 92 & 83 & -154 & 152 \\
\hline 16 & 45 & 45 & 47 & -100 & 98 & 89 & -162 & $160-162$ \\
\hline 17 & -48 & 46 & 49 & -106 & $102-106$ & 97 & -174 & 174 \\
\hline 19 & -52 & $48-50$ & 53 & -110 & 108 & & & \\
\hline
\end{tabular}

TABLE 1 . Old and new ranges for $N_{q}(4)$, for $q<100$.

But for every fixed genus $g>2$, nothing is known about the proportion of prime powers for which this bound is attained.

For $g=1$ and $g=2$, the exact value of $N_{q}(g)$ can be calculated. For $g=1$ this is due essentially to Deuring [4] (see [21, Thm. 4.1, p. 536]), and for $g=2$ to Serre 17 19] (see also 10 ). But even for $g=3$ difficulties arise, as is explained in 12 .

The online tables available at http://manypoints.org collect the best known upper and lower bounds on $N_{q}(g)$ for $g \leq 50$ and for various values of $q$ : the primes less than 100, the prime powers $p^{i}$ for $p<20$ and $i \leq 5$, and the powers of 2 up to $2^{7}$. Despite the lack of an explicit formula for $N_{q}(3)$, for all of the prime powers $q$ listed in these tables the value of $N_{q}(3)$ has been calculated. This leaves $N_{q}(4)$ as the next challenge. In this paper, we give new upper and lower bounds for $N_{q}(4)$ for 22 of the 23 prime powers $q<100$ for which the exact value had not been previously computed; we find the exact value of $N_{q}(4)$ for 17 of these prime powers.

Our results are given in Table 1. The entries in the 'old' column show the results that were listed on manypoints.org on 1 August 2011. Note that the entries in the 'new' column show that there are now only six values of $q$ less than 100 for which the exact value of $N_{q}(4)$ is not known.

We obtain our new upper bounds on $N_{q}(4)$ by using the results of [9]. For each $q$, we use the computer programs associated with that paper to obtain restrictions on genus- 4 curves over $\mathbf{F}_{q}$ with many points. Sometimes we learn that a curve with a certain number of points must be a double cover of one of several elliptic curves; in Section 3 we show how to enumerate such double covers. Sometimes we learn that a curve with a certain number of points must correspond to a Hermitian lattice over a quadratic order; we discuss these cases in Sections 4 and 5 . In two cases we find that the Jacobian of a curve with a certain number of points must have complex multiplication by $\mathbf{Z}\left[\zeta_{5}\right]$; our analysis of Hermitian forms over this ring 
in Section 6 6 shows that such curves do not exist. Our new lower bounds come from explicit examples of curves, which we present in Section 7 .

We have implemented all of our calculations in the computer algebra package Magma 2. As we mentioned above, we discover properties of genus-4 curves over $\mathbf{F}_{q}$ with a given number of points by using the programs associated with the paper [9. These programs are found in the package IsogenyClasses magma, which is available on the author's website: Go to the bibliography page

$$
\text { http://alumni.caltech.edu/ however/biblio.html }
$$

and follow the link associated with the paper $[\mathbf{9}$. The programs we use to enumerate double covers are also available online, by starting at the URL given above and following the link associated with the present paper.

\section{Restrictions on genus- 4 curves with many points}

In this section we present the results we obtained from running the programs in IsogenyClasses . magma.

Table 2 lists the $(q, N)$ pairs that we will have to eliminate in order to prove our new upper bounds; for each $q$ and $N$, the table shows what IsogenyClasses .magma tells us about genus- 4 curves over $\mathbf{F}_{q}$ with $N$ points. An entry of "None exist" means that IsogenyClasses.magma shows that no genus- 4 curve over $\mathbf{F}_{q}$ has exactly $N$ points. Entries of the form "Double cover of elliptic curve with trace $t$ " mean that any genus-4 curve over $\mathbf{F}_{q}$ with $N$ points must be a double cover of an elliptic curve over $\mathbf{F}_{q}$ with trace $t$. An entry of "Hermitian module over $R$ ", where $R$ is an order in an imaginary quadratic field, means that any genus- 4 curve over $\mathbf{F}_{q}$ with $N$ points must have a Jacobian that is isogenous to the fourth power of an ordinary elliptic curve over $\mathbf{F}_{q}$ whose Frobenius endomorphism generates the order $R$. An entry of "Hermitian module over $\mathbf{Z}\left[\zeta_{5}\right]$ " means that any genus- 4 curve over $\mathbf{F}_{q}$ with $N$ points must have a Jacobian that is isogenous to the square of an ordinary abelian surface with complex multiplication by the ring of integers of the 5th cyclotomic field.

\section{Double covers of elliptic curves}

Table 2 shows that there are a number of pairs $(q, N)$ such that a genus- 4 curve over $\mathbf{F}_{q}$ with $N$ points must be a double cover of an elliptic curve with a certain trace $t$. Thus, one way to show that there are no genus- 4 curves over $\mathbf{F}_{q}$ with $N$ points would be to enumerate all of the elliptic curve over $\mathbf{F}_{q}$ of trace $t$, enumerate all of the genus- 4 double covers of these curves, count the number of points on the double covers, and verify that none of them has $N$ points. This is the strategy we use for the appropriate entries in Table 2

In this section we explain more details of this procedure. The actual Magma programs we use can be found at the URL mentioned in the introduction; follow the link associated with this paper, and then download the file Genus4.magma.

Our first comment is that if $C$ is a double cover of an elliptic curve $E$ over a finite field $k$, and if $\sigma$ is an automorphism of $k$, then $C^{\sigma}$ is a double cover of $E^{\sigma}$. Since $C^{\sigma}$ and $C$ have the same number of points, it will suffice for us to enumerate all of the double covers of a set of representatives of the trace- $t$ elliptic curves up to automorphisms of $k$.

The finite fields we must investigate are small enough that we use a completely naive method of finding representatives for these elliptic curves. We will only be 


\begin{tabular}{|r|r|l|}
\hline$q$ & $N$ & Properties of a genus- 4 curve over $\mathbf{F}_{q}$ with $N$ points \\
\hline 11 & 34 & Hermitian module over $\mathbf{Z}\left[\zeta_{5}\right]$ \\
13 & 39 & Double cover of elliptic curve with trace -7 \\
17 & 48 & Double cover of elliptic curve with trace -8 \\
17 & 47 & None exist \\
19 & 52 & Hermitian module over $\mathbf{Z}[\sqrt{-3}]$ \\
19 & 51 & None exist \\
23 & 58 & Double cover of elliptic curve with trace -9 \\
29 & 70 & Hermitian module over $\mathbf{Z}[2 i]$ \\
29 & 69 & None exist \\
31 & 73 & Double cover of elliptic curve with trace -11 \\
37 & 84 & Double cover of elliptic curve with trace -12 \\
37 & 83 & None exist \\
41 & 90 & Hermitian module over $\mathbf{Z}[\sqrt{-5}]$ \\
41 & 89 & None exist \\
43 & 93 & Double cover of elliptic curve with trace -13 \\
47 & 100 & Hermitian module over $\mathbf{Z}[\alpha]$ \\
47 & 99 & None exist \\
53 & 110 & Hermitian module over $\mathbf{Z}[2 i]$ \\
53 & 109 & None exist \\
59 & 118 & Double cover of elliptic curve with trace -15 \\
59 & 117 & Double cover of elliptic curve with trace -15 \\
61 & 122 & Hermitian module over $\mathbf{Z}[\alpha]$ \\
61 & 121 & None exist \\
61 & 120 & Double cover of elliptic curve with trace -13, or \\
67 & 132 & Hermitian module over $\mathbf{Z}\left[\zeta_{5}\right]$ \\
67 & 131 & Hermitian module over $\mathbf{Z}[\sqrt{-3}]$ \\
67 & 130 & None exist \\
71 & 136 & Double cover of elliptic curve with trace -14 \\
71 & 135 & Hermitian module over $\mathbf{Z}[\sqrt{-7}]$ \\
73 & 139 & None exist \\
83 & 154 & Double cover of elliptic curve with trace -17 \\
83 & 153 & Double cover of elliptic curve with trace -18 \\
\end{tabular}

TABLE 2. What IsogenyClasses.magma tells us about genus-4 curves over $\mathbf{F}_{q}$ with $N$ points. Here $\zeta_{5}$ is a primitive 5 th root of unity, $i=\sqrt{-1}$, and $\alpha$ satisfies $\alpha^{2}+\alpha+5=0$. 
working with fields of characteristic larger than 3, so every elliptic curve can be written in the form $y^{2}=x^{3}+a x+b$, where $a$ and $b$ are elements of $k$ with $4 a^{3}+27 b^{2} \neq 0$. The curve determined by one such pair $(a, b)$ is isomorphic to a Galois conjugate of the curve determined by another pair $\left(a^{\prime}, b^{\prime}\right)$ if and only if $\left(a^{\prime}, b^{\prime}\right)=\left(a^{\sigma} u^{4}, b^{\sigma} u^{6}\right)$ for some $u \in k^{*}$ and some automorphism $\sigma$ of $k$. The function ECs takes as input a finite field $k$ and a trace $t$, explicitly computes the orbits of the set of $(a, b)$ pairs under the combined action of $k^{*}$ and Aut $k$, and computes the trace of one representative elliptic curve from each orbit. It returns those representatives that have the desired trace $t$.

Next, given an $E$ of trace $t$, we must enumerate its genus- 4 double covers $C$. We use the same general idea as in [8, §6.1]; our description of the method is adapted from the version given there.

The function field of such a $C$ is obtained from that of $E$ by adjoining a root of $z^{2}=f$, where $f$ is a function on $E$. By the Riemann-Hurwitz formula, in order for $C$ to have genus 4 the divisor of $f$ must be of the form

$$
P_{1}+\cdots+P_{6}+2 D,
$$

where the $P_{i}$ are distinct geometric points on $E$ such that the divisor $P_{1}+\cdots+P_{6}$ is $k$-rational, and where $D$ is a divisor of degree -3 . There is a function $g$ on $E$ such that

$$
D+\operatorname{div} g=Q-4 \infty,
$$

where $\infty$ is the infinite point on $E$ and where $Q$ is a rational point on $E$. Replacing $f$ with $f g^{2}$ gives an isomorphic double cover of $E$. Thus, we may assume that $C$ is given by adjoining a root of $z^{2}=f$, where $f$ is a function on $E$ whose divisor is of the form

$$
P_{1}+\cdots+P_{6}+2 Q-8 \infty, \quad \text { where the } P_{i} \text { are distinct. }
$$

We can also change the map $C \rightarrow E$ by following it with a translation map on $E$. Translating $E$ by a rational point $R$ has the effect of replacing $f$ with a function whose divisor is

$$
\left(P_{1}+R\right)+\cdots+\left(P_{6}+R\right)+2(Q+R)-8 R
$$

(where the sums in parentheses take place in the algebraic group $E$ ). By modifying this new $f$ by the square of a function we can get the divisor of $f$ to be

$$
\left(P_{1}+R\right)+\cdots+\left(P_{6}+R\right)+2(Q-3 R)-8 \infty .
$$

We see that we only need to consider $Q$ that represent distinct classes of $E(k)$ modulo $3 E(k)$. Note that every class other than the class of the identity element contains a representative that is not a 2 -torsion point, so we may assume that $Q$ does not have order 2. And finally, we note that we need only look at one representative from each orbit of Aut $E$ acting on $E(k) / 3 E(k)$.

Next, given an $E$ and a $Q \in E(k)$ not of order 2, we must enumerate all functions on $E$ with divisors of the form (3.1). Suppose first that $Q \neq \infty$. By translating coördinates on $E$, we may assume that $E$ is given by an equation $y^{2}=$ $x^{3}+r x^{2}+s x+t^{2}$ and that $Q$ is the point $(0, t)$. Since $Q$ is not a 2 -torsion point we have $t \neq 0$, and the tangent line to $E$ at $Q$ is given by $y=m x+t$, where $m=s /(2 t)$. Then there are two cases to consider: functions for which none of the $P_{i}$ is equal to $\infty$, and functions for which one of the $P_{i}$ is equal to $\infty$. 
If $f$ is a function on $E$ whose divisor has the desired form and for which no $P_{i}$ is $\infty$, then $f$ has degree 8 and lies in the Riemann-Roch space $\mathscr{L}(8 \infty-2 Q)$. We check that this space is spanned by the functions

$$
\left\{x^{4}, x^{2}(y-t), x^{3}, x(y-t), x^{2}, y-m x-t\right\} .
$$

We can then run through all of the $f$ 's in the $k$-span of these functions, considering only those linear combinations where the coefficient of $x^{4}$ is nonzero (so that the function actually has degree 8). In fact, since $z^{2}=f$ and $z^{2}=d^{2} f$ give isomorphic extensions of $E$, we can restrict our attention to linear combinations where the coefficient of $x^{4}$ is either 1 or a fixed nonsquare value.

For a given linear combination $f$, we can easily compute the number of points on the extension $z^{2}=f$ under the assumption that the divisor of $f$ is of the form (3.1). (We get either 2 or 0 points over $\infty$ depending on the leading term of the Laurent expansion of $f$ at $\infty$, and likewise for the points over $Q$; for the other points $P$ of $E(k)$, we get either 0,1 , or 2 points over $P$ depending on whether $f(P)$ is a nonsquare, zero, or a nonzero square.) If the number we calculate is larger than our previous best count, we can then check whether the divisor of $f$ is in fact of the form (3.1).

The case where one of the $P_{i}$ is equal to $\infty$ is very similar; the difference is that we now look in the Riemann-Roch space $\mathscr{L}(7 \infty-2 Q)$, and that now the extension $z^{2}=f$ always has exactly one point lying over $\infty$.

The function double_covers_genus_4 in the file Genus4.magma takes as input an elliptic curve $E$ over a finite field, and runs the algorithm sketched above to find the largest number of points on a genus-4 double cover of $E$. The function double_covers_given_trace takes as input a prime power $q$ and a trace $t$, and finds the maximal number of points on a genus- 4 curve over $\mathbf{F}_{q}$ that is a double cover of some elliptic curve over $\mathbf{F}_{q}$ of trace $t$.

For each pair $(q, N)$ whose associated entry in Table 2 says that a genus-4 curve over $\mathbf{F}_{q}$ with $N$ points must be a double cover of an elliptic curve of trace $t$, we ran our program with input $(q, t)$. For each pair, we found that the maximal number of points on a genus- 4 double cover of an elliptic curve with trace $t$ is less than $N$. Thus, for these pairs $(q, N)$, we find that $N_{q}(4)<N$.

\section{Hermitian forms over maximal quadratic orders}

The programs in IsogenyClasses.magma show that for several of the $(q, N)$ pairs listed in Table 2, every genus- 4 curve over $\mathbf{F}_{q}$ having $N$ points must have Jacobian isogenous to $E^{4}$, where $E$ is an ordinary elliptic curve over $\mathbf{F}_{q}$ whose Frobenius endomorphism generates a specific imaginary quadratic order $R$. The study of abelian varieties isogenous to $E^{4}$ is simplified by the use of Serre's 'Hermitian modules' [13. Appendix] or Deligne's equivalence of categories [3] between ordinary abelian varieties and modules over a certain ring, combined with the description of polarizations on these modules provided in [7.

In this section we analyze the three cases from Table 2 where the quadratic order in question is maximal; these are the cases $(q, N)=(41,90),(q, N)=(47,100)$, and $(q, N)=(61,122)$, where the corresponding quadratic order $\mathscr{O}$ has discriminant $-20,-19$, and -19 , respectively. As is explained in [13, Appendix], a principallypolarized abelian variety isogenous to $E^{4}$ corresponds to a principally-polarized 
Hermitian $\mathscr{O}$-module of rank 4. Schiemann 16 has computed all such principallypolarized Hermitian modules (up to isomorphism) for the quadratic orders we are concerned with; the lists can be found at

http://www.math.uni-sb.de/ag/schulze/Hermitian-lattices/ as well as on the author's web site, mentioned in the introduction.

Schiemann calculated the automorphism groups of these polarized Hermitian modules; these groups are isomorphic to the automorphism groups of the corresponding polarized abelian four-folds. Schiemann's tables show that for all of the Hermitian modules we must consider, the automorphism groups have order divisible by 4. By Torelli's theorem [15, Thm. 12.1, p. 202], if the polarized Jacobian of a curve $C$ has an automorphism group of order divisible by 4 , then either

- $C$ has an automorphism $\iota$ of order 2 such that the quotient curve $C_{0}=$ $C /\langle\iota\rangle$ has positive genus, or

- $C$ is a hyperelliptic curve with an automorphism of order 4 whose square is the hyperelliptic involution.

For the $(q, N)$ pairs we have to consider, we find that if $C$ is a genus- 4 curve over $\mathbf{F}_{q}$ with $N$ points, then either $C$ is a double cover of an elliptic curve isogenous to $E$, or $C$ is a double cover of a genus- 2 curve whose Jacobian is isogenous to $E^{2}$, or $C$ is a hyperelliptic curve with an automorphism whose square is the hyperelliptic involution.

We have already seen how to enumerate the genus- 4 double covers of an elliptic curve; running double_covers_given_trace for the $(q, t)$ pairs coming from our $(q, N)$ pairs, we find no genus- 4 curves over $\mathbf{F}_{q}$ with $N$ points. In Section 4.1 below we will show how to enumerate the genus- 4 double covers of genus- 2 curves, and in Section 4.2 we will show how to enumerate the genus- 4 hyperelliptic curves with an automorphism whose square is the hyperelliptic involution. For our $(q, N)$ pairs, we find no genus- 4 curves over $\mathbf{F}_{q}$ having $N$ points.

4.1. Double covers of genus- 2 curves. Suppose $\varphi: C \rightarrow C_{0}$ is a degree-2 map from a genus-4 curve to a genus- 2 curve over a finite field $k$ of characteristic greater than 2. The function field of $C$ is obtained from that of $C_{0}$ by adjoining a root of $z^{2}=f$, where $f$ is a function on $C_{0}$. By the Riemann-Hurwitz formula, in order for $C$ to have genus 4 the divisor of $f$ must be of the form

$$
P_{1}+P_{2}+2 D,
$$

where the $P_{i}$ are distinct geometric points on $E$ such that the divisor $P_{1}+P_{2}$ is $k$ rational, and where $D$ is a divisor of degree -1 . Let $\infty$ be any rational point on $C_{0}$. The Riemann-Roch theorem shows that the Riemann-Roch space $\mathscr{L}(D+4 \infty)$ has dimension 2 ; it follows that it must contain a function $g$ such that $\operatorname{div} g+D+3 \infty$ is effective. Then

$$
\operatorname{div} f g^{2}=P_{1}+P_{2}+2 D^{\prime}-6 \infty
$$

for some effective divisor $D^{\prime}$ of degree 2. Replacing $f$ with $f g^{2}$, we find that $C$ can be obtained from $C_{0}$ by adjoining a root of $z^{2}=f$, with $\operatorname{div} f=P_{1}+P_{2}+2 D^{\prime}-6 \infty$ for some effective $D^{\prime}$ of degree 2 .

Thus, to enumerate all genus- 4 curves that are double covers of $C_{0}$, we can simply enumerate all effective degree-2 divisors $D^{\prime}$, compute the Riemann-Roch space $\mathscr{L}\left(6 \infty-2 D^{\prime}\right)$, loop through the function $f$ in this space (up to squares in $k$ ) that are not squares in $\bar{k}\left(C_{0}\right)$, and consider the curves $z^{2}=f$. It is easy to count the number of points on a curve defined by such an extension. We implement this 
algorithm in the function double_covers_genus_4, the same function we used for doubles covers of elliptic curves; when the input to the function is a curve of genus 2, the function runs through the procedure sketched above, and outputs the largest number of points it finds.

For the case $(q, N)=(41,90)$ there are three possibilities for the base curve $C_{0}$; this can be determined by brute force (by enumerating all genus- 2 curves over $k$ ) or by theory, by noting that Schiemann's tables show exactly three unimodular rank-2 Hermitian $\mathscr{O}$-modules that are not products of two rank-1 modules. The three curves are

$$
\begin{aligned}
& y^{2}=x^{6}+7 x^{4}+8 x^{2}-7, \\
& y^{2}=x^{6}+7 x^{4}+3 x^{2}+7, \quad \text { and } \\
& y^{2}=x^{6}-3 x^{4}-3 x^{2}+1 .
\end{aligned}
$$

Running double_covers_genus_4 on these curves, we find no genus- 4 curves over $\mathbf{F}_{41}$ with 90 points. Similarly, we find one possible $C_{0}$ for each of the other two $(q, N)$ pairs we must consider. For $\mathbf{F}_{47}$ and $\mathbf{F}_{61}$, these curves are

$$
y^{2}=x^{6}+7 x^{4}-9 x^{2}-6 \text { and } y^{2}=x^{6}+10 x^{4}-11 x^{2}-1,
$$

respectively. Running double_covers_genus_4 on these curves, we find no genus-4 curves over $\mathbf{F}_{47}$ with 100 points, and no genus- 4 curves over $\mathbf{F}_{61}$ with 122 points.

4.2. Hyperelliptic curves with automorphisms of order 4. A hyperelliptic curve over $\mathbf{F}_{q}$ can have at most $2(q+1)$ rational points, so the only one of our three $(q, N)$ pairs that we need be concerned with is $(q, N)=(61,122)$.

Suppose $C$ is a genus- 4 hyperelliptic curve over a finite field $k$ with an automorphism $\alpha$ of order 4 whose square is the hyperelliptic involution. (In fact, 5 , Thm. 1.1] shows that every order- 4 automorphism of a genus- 4 hyperelliptic curve has square equal to the hyperelliptic involution.) We can choose a parameter for $\mathbf{P}^{1}$ so that the automorphism of $\mathbf{P}^{1}$ induced by $\alpha$ is $x \mapsto a / x$ for some $a \in k^{*}$.

Writing $C$ as $y^{2}=f$ for some separable polynomial $f \in k[x]$ of degree 9 or 10 , we see that the automorphism $\alpha$ must have the form $(x, y) \mapsto\left(a / x, b y / x^{5}\right)$, and since $\alpha^{2}$ is the hyperelliptic involution we must have $b^{2}=-a^{5}$. Replacing $x$ with $a^{2} x / b$, we compute that $\alpha$ is given by $(x, y) \mapsto\left(-1 / x, y / x^{5}\right)$.

We find that $f$ must be a linear combination of the following polynomials:

$$
\left\{x^{10}+1, x^{9}-x, x^{8}+x^{2}, x^{7}-x^{3}, x^{6}+x^{4}\right\} .
$$

For the one pair $(q, N)$ we must consider, we can examine all linear combinations of these polynomials (up to squares in $\mathbf{F}_{q}$ ), and compute the number of points on the associated hyperelliptic curves. We find no curve over $\mathbf{F}_{61}$ having 122 points.

The function hyperelliptic in the file Genus4.magma implements this algorithm.

\section{Hermitian forms over nonmaximal quadratic orders}

For five of the entries $(q, N)$ in Table 2 we see that the Jacobian of a genus-4 curve over $\mathbf{F}_{q}$ with $N$ points must be isogenous to $E^{4}$, where $E$ lies in an isogeny class of ordinary elliptic curves over $\mathbf{F}_{q}$ all of whose elements have complex multiplication by a non-maximal order $R$ in a quadratic field $K$; here $R$ is the ring generated over $\mathbf{Z}$ by the Frobenius endomorphism of $E$. The five entries are $(19,52),(29,70)$, $(53,110),(67,132)$, and $(71,136)$, and the rings $R$ are the orders of conductor 2 
inside the maximal orders of, respectively, $\mathbf{Q}(\sqrt{-3}), \mathbf{Q}(i), \mathbf{Q}(i), \mathbf{Q}(\sqrt{-3})$, and $\mathbf{Q}(\sqrt{-7})$. The discriminants of the rings $R$ are, respectively, $-12,-16,-16,-12$, and -28 . We will show that for each of these pairs $(q, N)$, a genus- 4 curve over $\mathbf{F}_{q}$ having $N$ points must be a double cover of a curve of genus 1 or 2 .

Proposition 5.1. Let $t$ be the trace of Frobenius for an isogeny class $\mathscr{C}$ of ordinary elliptic curves over a finite field $\mathbf{F}_{q}$ such that $\Delta:=t^{2}-4 q$ lies in $\{-12,-16,-28\}$. Suppose $C$ is a genus-4 curve over $\mathbf{F}_{q}$ whose Jacobian is isogenous to the fourth power of an elliptic curve in $\mathscr{C}$.

(1) If $\Delta=-12$ or $\Delta=-16$ then $C$ is a double cover of a curve in $\mathscr{C}$.

(2) If $\Delta=-28$ then either $C$ is a double cover of a curve in $\mathscr{C}$, or $C$ is a double cover of a genus-2 curve whose Jacobian is isogenous to the square of a curve in $\mathscr{C}$.

For our five $(q, N)$ pairs, the function double_covers_given_trace finds no genus- 4 curve with $N$ points. For the pair $(q, N)=(71,136)$ we must also run double_covers_genus_4 on three genus- 2 curves:

$$
\begin{aligned}
& y^{2}=x^{6}-29 x^{4}-29 x^{2}+1, \\
& y^{2}=x^{6}-13 x^{4}-13 x^{2}+1, \quad \text { and } \\
& y^{2}=x^{6}-12 x^{4}-15 x^{2}-1 .
\end{aligned}
$$

Again, we find no genus-4 curve with $N$ points.

The proof of Proposition 5.1 relies on several lemmas. To begin, we show that for each of our five cases, there are only four abelian varieties to consider.

Lemma 5.2. Let $t$ be the trace of Frobenius for an isogeny class $\mathscr{C}$ of ordinary elliptic curves over a finite field $\mathbf{F}_{q}$ such that $\Delta:=t^{2}-4 q$ lies in $\{-12,-16,-28\}$. Then $\mathscr{C}$ contains exactly two elliptic curves, one of them with endomorphism ring equal to the maximal order $\mathscr{O}$ in $K:=\mathbf{Q}(\sqrt{\Delta})$, and one with endomorphism ring equal to the order $R$ of conductor 2 in $\mathscr{O}$.

Let $E$ and $F$ be these two elliptic curves, where $E$ has the larger endomorphism ring. Then every abelian variety over $\mathbf{F}_{q}$ isogenous to $E^{n}$ is isomorphic to $E^{i} \times F^{n-i}$ for some $i$, and the varieties arising from distinct values of $i$ are not isomorphic to one another.

Proof. By Deligne's equivalence of categories [3, the elliptic curves in $\mathscr{C}$ correspond to the isomorphism classes of nonzero finitely-generated $R$-modules in $K$. The endomorphism ring of such a module is either $R$ or $\mathscr{O}$, and since both $R$ and $\mathscr{O}$ have class number 1 , there is one elliptic curve $E$ with End $E \cong \mathscr{O}$ and one elliptic curve $F$ with End $F \cong R$.

Likewise, Deligne's theorem shows that the varieties isogenous to $E^{n}$ correspond to rank- $n$ modules over $R$. By using a result of Borevich and Faddeev [1, we see that every such module is isomorphic to a sum of copies of $\mathscr{O}$ plus a sum of copies of $R$, and that two such modules are isomorphic if and only if they have the same number of each type of summand. The second statement of the lemma follows upon applying Deligne's equivalence of categories.

Next, we show that for each of our cases, any curve with Jacobian isomorphic to $E^{i} \times F^{4-i}$ with $i>0$ must be a double cover of $E$. 
LEMma 5.3. Let $t$ be the trace of Frobenius for an isogeny class of ordinary elliptic curves over a finite field $\mathbf{F}_{q}$ such that $\Delta:=t^{2}-4 q$ lies in $\{-12,-16,-28\}$, and let $E$ and $F$ be as in Lemma 5.2. If $C$ is a genus-4 curve over $\mathbf{F}_{q}$ whose Jacobian is isomorphic to $E^{i} \times F^{4-i}$ for some $i>0$, then $C$ is a double cover of $E$.

Proof. Our proof is computational, and uses the function pullback_bound from the package IsogenyClasses .magma. Given a fundamental discriminant $D$ with $-11 \leq D<0$, an integer $n$, and a dimension $d$, this function will return an integer $B$ such that for every $d \times d$ Hermitian matrix of determinant $n$ with entries in the quadratic order $\mathscr{O}_{D}$ of discriminant $D$, the Hermitian form on $\mathscr{O}_{D}^{d}$ determined by this matrix will have a nonzero vector of length at most $B$. As is explained in $[9, \S 4]$, this translates into a statement about polarizations on $E^{d}$, where $E$ has complex multiplication by $\mathscr{O}_{D}$; namely, for every degree- $n^{2}$ polarization $\lambda$ on $E^{d}$, there is an embedding $\varphi: E \rightarrow E^{d}$ such that $\varphi^{*} \lambda$ is a polarization on $E$ of degree at most $B^{2}$.

Now suppose $C$ is a curve with Jacobian isomorphic to $E^{i} \times F^{4-i}$, with $i>0$, and let $\lambda$ be the canonical principal polarization on $\operatorname{Jac} C$. There is an isogeny of degree $2^{4-i}$ from $E^{4}$ to Jac $C$, and pulling back $\lambda$ via this isogeny gives a polarization $\mu$ of degree $4^{4-i}$ on $E^{4}$. The function pullback_bound tells us that we can pull back $\mu$ to a polarization of degree 1 or 4 on $E$, and by $[9$, Lem. 4.3] this gives us a map of degree 1 or 2 from $C$ to $E$. A map of degree 1 is clearly impossible, so we find that $C$ is a double cover of $E$.

We see that to prove Proposition 5.1 we need only consider curves whose Jacobians are isomorphic to $F^{4}$; in other words, we need only consider principal polarizations on $F^{4}$. For $\Delta=-12$ and $\Delta=-16$ we will show that every such polarization can be pulled back to either $E$ or $F$ to get a polarization of degree 1 or 4 . For $\Delta=-28$ we will show that if such a polarization cannot be pulled back to a polarization of degree 1 or 4 on $E$ or $F$, and if the polarized variety is the Jacobian of a curve, then the curve has an involution that makes it a double cover of a genus-2 curve.

Let $\varphi$ be a degree-2 isogeny from $E$ to $F$, and let $\Phi: E^{4} \rightarrow F^{4}$ be the degree-16 product isogeny $\varphi \times \varphi \times \varphi \times \varphi$. Denote the kernel of $\Phi$ by $G$. Note that the smallest $\mathscr{O}$-stable subgroup of $E^{4}$ that contains $G$ is $E^{4}[2]$.

Suppose $\lambda$ is a principal polarization on $F^{4}$, and let $\mu=\Phi^{*} \lambda$ be the pullback of $\lambda$ to $E^{4}$. Then $\mu$ is a polarization of degree $16^{2}$ on $E^{4}$, and since $\operatorname{ker} \mu$ is stable under the action of $\mathscr{O}$ and contains $G$, we must have $\operatorname{ker} \mu=E^{4}[2]$. That means that $\mu$ must be twice a principal polarization.

Thus, to enumerate the possible principal polarizations $\lambda$ on $F^{4}$, we can enumerate the principal polarizations on $E^{4}$ up to isomorphism, multiply each of these by 2 to get a polarization $\mu$ of degree $16^{2}$ on $E^{4}$, enumerate the subgroups $G$ of $E^{4}[2]$ that generate all of $E^{4}[2]$ as an $\mathscr{O}$-module and that are isotropic with respect to the Weil pairing on $E^{4}[2]$ determined by $\mu$, and then compute the matrix in $M_{4}(R)$ that represents the polarization we get by pushing $\mu$ down through an isogeny with kernel $G$. The Magma programs we use to do this can be found at the URL mentioned in the introduction; follow the link associated with this paper, and then download the file NonMaximalOrders.magma.

5.1. Proof of Proposition 5.1 when $\Delta=-12$. Schiemann's tables [16] show that up to isomorphism the only principal polarization on $E^{4}$ is the product 
polarization, so our only $\mu$ is represented by the diagonal matrix with 2's on the diagonal. Embedding $E$ into $E^{4}$ along one of the factors, we find that $\mu$, and hence $\lambda$, can be pulled back to a polarization of degree 4 on $E$.

5.2. Proof of Proposition 5.1 when $\Delta=-16$. Schiemann's tables show two principal polarizations on $E^{4}$, the product polarization and the polarization given by the Hermitian matrix

$$
P=\left[\begin{array}{cccc}
2 & 1 & 0 & 0 \\
1 & 2 & 1-i & 1-i \\
0 & 1+i & 2 & 1 \\
0 & 1+i & 1 & 2
\end{array}\right]
$$

Twice the product polarization can be pulled back to give a polarization of degree 4 on $E$, so we need only consider the polarization $2 P$.

A computer calculation shows that there are 1024 subgroups of $E^{4}[2]$ that are maximal isotropic with respect to the Weil pairing determined by $P$ and that generate all of $E^{4}[2]$ as an $\mathscr{O}$-module. For each such subgroup $G$, we compute the polarization on $F^{4}$ obtained by pushing the polarization $2 P$ down through the isogeny with kernel $G$; this polarization can be represented by a Hermitian matrix in $M_{4}(R)$, which gives a Hermitian form on $R^{4}$. We can then compute the short vectors in this Hermitian lattice. We find that for each group $G$, the Hermitian lattice has a short vector of length 2 . This means that the polarization on $F^{4}$ can be pulled back to a polarization of degree 4 on $F$.

5.3. Proof of Proposition 5.1 when $\Delta=-28$. Schiemann's tables show that there are three principal polarizations on $E^{4}$ : the product polarization and the polarizations given by the Hermitian matrices

$$
P_{1}=\left[\begin{array}{cccc}
1 & 0 & 0 & 0 \\
0 & 2 & \frac{1+\sqrt{-7}}{2} & -1 \\
0 & \frac{1-\sqrt{-7}}{2} & 2 & \frac{1+\sqrt{-7}}{2} \\
0 & -1 & \frac{1-\sqrt{-7}}{2} & 2
\end{array}\right]
$$

and

$$
P_{2}=\left[\begin{array}{cccc}
2 & 1 & 0 & \frac{1+\sqrt{-7}}{2} \\
1 & 2 & \frac{1+\sqrt{-7}}{2} & \frac{1+\sqrt{-7}}{2} \\
0 & \frac{1-\sqrt{-7}}{2} & 2 & 1 \\
\frac{1-\sqrt{-7}}{2} & \frac{1-\sqrt{-7}}{2} & 1 & 2
\end{array}\right] .
$$

There is a 2 on the diagonal of $2 P_{1}$, so this polarization can be pulled back to a degree- 4 polarization on $E$. Likewise, twice the product polarization can be pulled back to a degree- 4 polarization on $E$. That leaves us to consider the polarization $2 P_{2}$.

A computer calculation shows that there are 448 subgroups of $E^{4}[2]$ that are maximal isotropic with respect to the Weil pairing determined by $P_{2}$ and that generate all of $E^{4}[2]$ as an $\mathscr{O}$-module. Of these subgroups, 256 give rise to principal polarizations on $F^{4}$ whose associated Hermitian forms have short vectors of length 2. The other 192 subgroups give principal polarizations on $F^{4}$ that are 
isomorphic to the polarizations defined by one of the following three Hermitian matrices:

$$
\begin{aligned}
Q_{1} & =\left[\begin{array}{cccc}
4 & 2 & 2 & -\sqrt{-7} \\
2 & 4 & -\sqrt{-7} & 2 \\
2 & \sqrt{-7} & 4 & -2 \\
\sqrt{-7} & 2 & -2 & 4
\end{array}\right] \\
Q_{2} & =\left[\begin{array}{ccccc}
3 & 1 & 1 & -1-\sqrt{-7} \\
1 & 3 & -1-\sqrt{-7} & 1 \\
1 & -1+\sqrt{-7} & 4 & -2 \\
-1+\sqrt{-7} & 1 & -2 & 4
\end{array}\right] \\
Q_{3} & =\left[\begin{array}{cccc}
3 & 1 & 0 & -\sqrt{-7} \\
1 & 3 & -\sqrt{-7} & 0 \\
0 & \sqrt{-7} & 3 & -1 \\
\sqrt{-7} & 0 & -1 & 3
\end{array}\right] .
\end{aligned}
$$

We check that for each of these three polarizations $Q$ there is an involution of the polarized variety $\left(F^{4}, Q\right)$ that fixes a 2-dimensional subvariety of $F^{4}$; such an involution can be represented by a matrix $A$ such that $A^{2}=I$ and $A^{*} Q A=Q$, and such that $A-I$ has rank 2 . For each $Q$ we note that the matrix

$$
A=\left[\begin{array}{llll}
0 & 1 & 0 & 0 \\
1 & 0 & 0 & 0 \\
0 & 0 & 0 & 1 \\
0 & 0 & 1 & 0
\end{array}\right]
$$

has these properties. It follows from Torelli's theorem [15. Thm. 12.1, p. 202] that if $\left(F^{4}, Q\right)$ is the Jacobian of a curve $C$, then $C$ has an involution $\alpha$ such that the quotient of $C$ by $\alpha$ is a genus-2 curve whose Jacobian is necessarily isogenous to $F^{2}$.

This completes the proof of Proposition 5.1

\section{Hermitian forms over $\mathbf{Z}\left[\zeta_{5}\right]$}

Let $\mathscr{O}=\mathbf{Z}\left[\zeta_{5}\right]$. As Table 2 indicates, the programs in IsogenyClasses .magma show that if $C$ is a genus- 4 curve over $\mathbf{F}_{11}$ with 34 points, the center of the endomorphism ring of the Jacobian of $C$ is isomorphic to $\mathscr{O}$. (At the end of this section we will explain how to derive this statement from the output of the programs.) Likewise, if $C$ is a genus- 4 curve over $\mathbf{F}_{61}$ with 120 points that is not a double cover of an elliptic curve, then the center of the endomorphism ring of its Jacobian is isomorphic to $\mathscr{O}$. In this section, we show that no ordinary genus- 4 curve over a finite field can have $\mathscr{O}$ as the center of the endomorphism ring of its Jacobian.

Proposition 6.1. Let $k$ be a finite field. There is no genus-4 curve over $k$ with ordinary Jacobian $J$ such that the center of End $J$ is isomorphic to $\mathbf{Z}\left[\zeta_{5}\right]$.

Proof. Suppose, to obtain a contradiction, that such a curve $C$ exists. Let $K=\mathbf{Q}\left(\zeta_{5}\right)$, let $\pi$ be the Frobenius endomorphism of $J$, let $\bar{\pi}$ be the Verschiebung endomorphism of $J$, and let $R$ be the ring $\mathbf{Z}[\pi, \bar{\pi}]$. Since $R$ lies in the center of End $J$ we may view $R$ as a subring of $\mathscr{O}$, and since $\pi$ generates the center of $($ End $J) \otimes \mathbf{Q}$, we see that $R$ is an order in $\mathscr{O}$. Let $M$ be the Deligne module associated with $J$ (see [7), so that $M$ is a finitely-generated $R$-module that is isomorphic to a submodule of $K^{2}$. Since End $J$ contains $\mathscr{O}$, we see that $M$ is in fact an $\mathscr{O}$-module, 
and since $\mathscr{O}$ has class number 1 we have $M \cong \mathscr{O} \oplus \mathscr{O}$. Translating this statement back from Deligne modules to ordinary abelian varieties, we see that $J \cong A \times A$ for a 2-dimensional abelian variety with End $A \cong \mathscr{O}$.

The field $K$ is ramified over its real subfield at a finite prime, so by $\mathbf{7}$, Cor. 11.4, p. 2391] the variety $A$ has a principal polarization $\lambda$. Let $\mu$ be the canonical polarization on $J$, viewed as a polarization on $A \times A$. Then $\mu$ is equal to the product polarization $\lambda \times \lambda$ preceded by an endomorphism $P$ of $A \times A$; viewing $P$ as an element of $M_{2}(\mathscr{O})$, we find that $P$ is a unimodular Hermitian matrix that is totally positive (meaning that all of the roots of its minimal polynomial are totally positive algebraic numbers). But Lemma 6.2 below says that all totally positive rank-2 unimodular Hermitian lattices over $\mathscr{O}$ are decomposable, so $\mu$ is isomorphic to the product polarization $\lambda \times \lambda$. This contradicts the fact that the polarization on a Jacobian is never a product.

Lemma 6.2. Let $\mathscr{O}=\mathbf{Z}\left[\zeta_{5}\right]$. Suppose $P$ is a totally positive unimodular Hermitian matrix in $M_{2}(\mathscr{O})$. Then there is an invertible $C \in M_{2}(\mathscr{O})$ such that $P=C^{*} C$, where $C^{*}$ is the conjugate transpose of $C$.

Proof. Our proof follows the lines set out in $[\mathbf{8}, \S 8]$.

Let $K=\mathbf{Q}\left(\zeta_{5}\right)$, let $\varphi$ be a fundamental unit of the maximal real subfield $K^{+}=\mathbf{Q}(\sqrt{5})$ of $K$ with $\operatorname{Tr}_{K^{+} / \mathbf{Q}} \varphi=1$, and let $\phi$ be the real number $(1+\sqrt{5}) / 2$. Let $\psi_{1}$ and $\psi_{2}$ be embeddings of $K$ into the complex numbers $\mathbf{C}$ with $\psi_{1}(\varphi)=\phi$ and $\psi_{2}(\varphi)=1 / \phi$. If $z$ is a complex number, we let $|z|$ be its magnitude and we let $\|z\|$ be its norm, so that $\|z\|=|z|^{2}=z \bar{z}$.

Let $q$ be the quadratic form on $\mathscr{O}$ that sends $x$ to the trace from $K$ to $\mathbf{Q}$ of $x \bar{x}$, so that $q(x)=2\left\|\psi_{1}(x)\right\|+2\left\|\psi_{2}(x)\right\|$. Let $\Lambda$ be the lattice $(\mathscr{O}, q)$. Using Magma, we compute the Voronoi cell for this lattice, and we find that the covering radius of the lattice is 2 ; that is, every element of $\Lambda \otimes \mathbf{Q}$ differs from a lattice point by an element $x$ with $q(x) \leq 2$. This means that every element of $K$ differs from an element of $\mathscr{O}$ by an element $x$ satisfying

$$
\left\|\psi_{1}(x)\right\|+\left\|\psi_{2}(x)\right\| \leq 1 .
$$

In particular, we see that this $x$ also satisfies

$$
N_{K / \mathbf{Q}}(x) \leq 1 / 4 \quad \text { and } \quad\left\|\psi_{i}(x)\right\| \leq 1 \quad \text { for } i=1,2 .
$$

In turn, this statement about the lattice $\Lambda$ gives us a Euclidean algorithm on $\mathscr{O}$ : Given elements $n$ and $d$ of $\mathscr{O}$, there are elements $q$ and $r$ of $\mathscr{O}$ such that $n=q d+r$ with

$$
N(r) \leq 1 / 4 \quad \text { and } \quad\left\|\psi_{i}(r)\right\| \leq\left\|\psi_{i}(n)\right\| \quad \text { for } i=1,2 .
$$

Write our totally positive unimodular Hermitian matrix $P$ as

$$
P=\left[\begin{array}{ll}
\alpha & \bar{\beta} \\
\beta & \gamma
\end{array}\right]
$$

where $\alpha$ and $\gamma$ are totally real and where $\alpha$ and $\alpha \gamma-\beta \bar{\beta}$ are totally positive. We will repeatedly choose invertible matrices $C$ and replace $P$ with $C^{*} P C$ in order to reduce the size of the norm of the upper left element of $P$.

The determinant of $P$ is a totally positive unit in $K^{+}$, and so is an even power of $\varphi$. By modifying $P$ by a matrix $C$ of the form

$$
\left[\begin{array}{cc}
\varphi^{i} & 0 \\
0 & 1
\end{array}\right]
$$


we may assume that $P$ has determinant 1 . Then by modifying $P$ by a power of the matrix

we can ensure that

$$
\left[\begin{array}{cc}
\varphi & 0 \\
0 & \varphi^{-1}
\end{array}\right]
$$

$$
\frac{1}{\phi^{2}} \leq \frac{\psi_{1}(\alpha)}{\psi_{2}(\alpha)} \leq \phi^{2}
$$

Another way of expressing this is to say that

$$
\frac{1}{\phi^{2}} \leq \frac{\psi_{i}(\alpha)^{2}}{\operatorname{Norm}_{K^{+} / \mathbf{Q}}(\alpha)} \leq \phi^{2} \quad \text { for } i=1,2 .
$$

Applying our Euclidean algorithm to $\beta$ and $\alpha$, we find that $\beta=q \alpha+r$ for a $q$ and an $r$ with $\left\|\psi_{i}(r)\right\| \leq\left\|\psi_{i}(\alpha)\right\|$ and with $\operatorname{Norm}_{K / \mathbf{Q}}(r) \leq(1 / 4) \operatorname{Norm}_{K / \mathbf{Q}}(\alpha)$. If we set

then

$$
C=\left[\begin{array}{cc}
1 & -\bar{q} \\
0 & 1
\end{array}\right]
$$

$$
C^{*} P C=\left[\begin{array}{cc}
\alpha & \bar{r} \\
r & \gamma^{\prime}
\end{array}\right]
$$

for some integer $\gamma^{\prime}$ in $K^{+}$. Replace $\beta$ with $r$ and $\gamma$ with $\gamma^{\prime}$, so that now we have

$$
\left\|\psi_{i}(\beta)\right\| \leq\left\|\psi_{i}(\alpha)\right\| \quad \text { for } i=1,2
$$

and

$$
\operatorname{Norm}_{K / \mathbf{Q}}(\beta) \leq(1 / 4) \operatorname{Norm}_{K / \mathbf{Q}}(\alpha)
$$

Let $B=\beta \bar{\beta}$, so that $B$ is an integer in $K^{+}$. Note that we have $\alpha \gamma-B=1$, so

$$
\psi_{i}(\alpha) \psi_{i}(\gamma)=1+\psi_{i}(B) \quad \text { for } i=1,2
$$

and therefore

$$
\psi_{i}(\gamma) / \psi_{i}(\alpha)=1 / \psi_{i}(\alpha)^{2}+\psi_{i}(B) / \psi_{i}(\alpha)^{2} \quad \text { for } i=1,2 .
$$

Now let

$$
\begin{aligned}
& b_{1}=\psi_{1}(B) / \psi_{1}\left(\alpha^{2}\right) \\
& b_{2}=\psi_{2}(B) / \psi_{2}\left(\alpha^{2}\right) \\
& c_{1}=1 / \psi_{1}\left(\alpha^{2}\right) \\
& c_{2}=1 / \psi_{2}\left(\alpha^{2}\right)
\end{aligned}
$$

so that equation (6.4) becomes

$$
\psi_{1}(\gamma) / \psi_{1}(\alpha)=b_{1}+c_{1} \quad \text { and } \quad \psi_{2}(\gamma) / \psi_{2}(\alpha)=b_{2}+c_{2}
$$

Multiplying these last two equalities gives

$$
\operatorname{Norm}_{K^{+} / \mathbf{Q}}(\gamma / \alpha)=b_{1} b_{2}+b_{1} c_{2}+b_{2} c_{1}+c_{1} c_{2} .
$$

Note that

$$
b_{1} b_{2}=\frac{\operatorname{Norm}_{K^{+} / \mathbf{Q}}(B)}{\operatorname{Norm}_{K^{+} / \mathbf{Q}}(\alpha)^{2}}=\frac{\operatorname{Norm}_{K / \mathbf{Q}}(\beta)}{\operatorname{Norm}_{K / \mathbf{Q}}(\alpha)} \leq \frac{1}{4}
$$

(where the final inequality comes from (6.3)) and

$$
c_{1} c_{2}=\frac{1}{\operatorname{Norm}_{K^{+} / \mathbf{Q}}\left(\alpha^{2}\right)} \text {. }
$$


Furthermore, from inequality (6.1) we see that

$$
c_{1} \leq \frac{\phi^{2}}{\operatorname{Norm}_{K^{+} / \mathbf{Q}}(\alpha)} \quad \text { and } \quad c_{2} \leq \frac{\phi^{2}}{\operatorname{Norm}_{K^{+} / \mathbf{Q}}(\alpha)},
$$

and from inequality (6.2) we see that

$$
b_{1}=\frac{\left\|\psi_{1}(\beta)\right\|}{\left\|\psi_{1}(\alpha)\right\|} \leq 1 \quad \text { and } \quad b_{2}=\frac{\left\|\psi_{2}(\beta)\right\|}{\left\|\psi_{2}(\alpha)\right\|} \leq 1 .
$$

If we view $b_{1}, b_{2}, c_{1}$, and $c_{2}$ as non-negative real variables subject only to the conditions expressed in relations (6.6), (6.7), (6.8), and (6.9), and if we maximize $b_{1} c_{1}+b_{2} c_{2}$ subject to these conditions, we find that the maximum value occurs when $b_{1}=1$ and $c_{1}=\phi^{2} / \operatorname{Norm}_{K^{+} / \mathbf{Q}}(\alpha)$. Thus we have

$$
b_{1} c_{1}+b_{2} c_{2} \leq 1 \cdot \frac{\phi^{2}}{\operatorname{Norm}_{K^{+} / \mathbf{Q}}(\alpha)}+\frac{1}{4} \cdot \frac{\left(1 / \phi^{2}\right)}{\operatorname{Norm}_{K^{+} / \mathbf{Q}}(\alpha)} \leq \frac{2.72}{\operatorname{Norm}_{K^{+} / \mathbf{Q}}(\alpha)} .
$$

Let $\epsilon=1 / \operatorname{Norm}_{K^{+} / \mathbf{Q}}(\alpha)$. Then by combining the relations (6.5), (6.6), (6.7) and (6.10) we find that

$$
\operatorname{Norm}_{K+/ \mathbf{Q}}(\gamma / \alpha) \leq \epsilon^{2}+2.72 \epsilon+1 / 4
$$

If $\operatorname{Norm}_{K^{+} / \mathbf{Q}}(\alpha) \geq 4$ then $\epsilon \leq 1 / 4$ and $\operatorname{Norm}_{K^{+} / \mathbf{Q}}(\gamma / \alpha)<1$. Then we can modify $P$ by

$$
\left[\begin{array}{ll}
0 & 1 \\
1 & 0
\end{array}\right]
$$

to exchange $\alpha$ and $\gamma$, and this decreases the norm of the upper left element of $P$.

We repeat this procedure until we reach the point where $\operatorname{Norm}_{K^{+} / \mathbf{Q}}(\alpha) \leq 3$. The only totally positive integer of $K^{+}$with norm less than 4 is 1 , so $\alpha=1$ and we can reduce $\beta$ to be 0 . Then we find $\gamma=1$, so that we have reduced $P$ to the identity matrix.

Let us turn to the specific pairs $(q, N)$ that we must consider. For $q=11$ and $N=34$, the function isogeny_classes in the package IsogenyClasses.magma shows that a genus- 4 curve $C$ over $\mathbf{F}_{11}$ with 34 points must have real Weil polynomial equal to $\left(x^{2}+11 x+29\right)^{2}$; that is, the characteristic polynomial of Frobenius plus Verschiebung is the square of this polynomial. It follows that the characteristic polynomial of Frobenius is

$$
\left(x^{4}+11 x^{3}+51 x^{2}+121 x+121\right)^{2},
$$

which means that the Jacobian of $C$ is isogenous to the square of an abelian surface $A$ with characteristic polynomial $x^{4}+11 x^{3}+51 x^{2}+121 x+121$. Since the middle coefficient of this characteristic polynomial is coprime to $q=11$, we see that $A$ is ordinary. Furthermore, the polynomial has a root $\pi$ in $\mathbf{Q}\left(\zeta_{5}\right)$, namely $\pi=$ $\zeta_{5}^{2}+2 \zeta_{5}-2$. One checks that $\pi$ and its complex conjugate generate the ring $\mathbf{Z}\left[\zeta_{5}\right]$, so the center of End $J$, which contains Frobenius and Verschiebung, must equal $\mathbf{Z}\left[\zeta_{5}\right]$. As we have seen, this is impossible.

For $q=61$ and $N=120$, the function isogeny_classes tells us that if a genus4 curve $C$ over $\mathbf{F}_{61}$ with 120 points is not a double cover of an elliptic curve of trace -13 , then it must have real Weil polynomial equal to $\left(x^{2}+29 x+209\right)^{2}$. As above, we see that this implies that $C$ is ordinary and the center of the endomorphism ring of its Jacobian is $\mathbf{Z}\left[\zeta_{5}\right]$, which is impossible. 


\begin{tabular}{|r|r|ll|}
\hline$q$ & $N$ & Equations for a genus-4 curve over $\mathbf{F}_{q}$ with $N$ points \\
\hline 13 & 38 & $y^{2}=x^{3}+4$ & $z^{2}=x^{3}+x^{2}-4 x-3$ \\
17 & 46 & $y^{2}=x^{3}+x+8$ & $z^{2}=x^{3}-5 x^{2}-2 x-8$ \\
19 & 48 & $y^{2}=x^{3}+8$ & $z^{2}=x^{3}-9 x^{2}-5$ \\
23 & 57 & $y^{2}=x^{3}-6 x^{2}-3 x-7$ & $z^{2}=x y+2 x^{3}-11 x^{2}+7 x+1$ \\
29 & 67 & $y^{2}=x^{3}-3 x+18$ & $z^{2}=y+7 x^{3}+6 x^{2}+2 x-10$ \\
31 & 72 & $y^{2}=x^{3}+x+10$ & $z^{2}=x^{3}+7 x^{2}-13 x-13$ \\
37 & 82 & $y^{2}=x^{3}+2 x$ & $z^{2}=x^{3}+x^{2}-10 x-13$ \\
41 & 88 & $y^{2}=x^{3}+6 x+5$ & $z^{2}=3 x^{3}-17 x^{2}-11 x$ \\
43 & 92 & $y^{2}=x^{3}+2 x+1$ & $z^{2}=x^{3}-6 x^{2}+11 x$ \\
47 & 98 & $y^{2}=x^{5}-6 x^{3}+8 x^{2}-5 x+12$ & $z^{2}=y+6 x^{3}+6 x^{2}-x-3$ \\
49 & 102 & $y^{2}=x^{3}+x$ & $z^{2}=x^{3}+x^{2}+x+4$ \\
53 & 108 & $y^{2}=x^{3}+4 x+10$ & $z^{2}=x^{3}+12 x^{2}+17 x+9$ \\
59 & 116 & $y^{2}=x^{3}+2 x+22$ & $z^{2}=2 x^{3}+x^{2}-x+9$ \\
61 & 118 & $y^{2}=x^{3}+4$ & $z^{2}=x^{3}+23 x^{2}+25 x+36$ \\
67 & 129 & $y^{2}=x^{3}+25$ & $z^{2}=x y-8 x^{3}-27 x+4$ \\
71 & 134 & $y^{2}=x^{3}+x+9$ & $z^{2}=x^{3}+9 x^{2}+24 x-9$ \\
73 & 138 & $y^{2}=x^{3}+3 x+11$ & $z^{2}=x^{3}+34 x^{2}+18 x+40$ \\
79 & 148 & $y^{2}=x^{3}+x+6$ & $z^{3}=y+33 x+2$ \\
83 & 152 & $y^{2}=x^{3}+2 x+19$ & $z^{2}=x^{3}+38 x^{2}-6 x+39$ \\
89 & 160 & $y^{2}=x^{3}+3 x$ & $z^{2}=x^{3}+13 x^{2}-22 x+28$ \\
97 & 174 & $y^{2}=x^{3}+5 x+26$ & $z^{3}=y+37 x+16$ \\
\hline
\end{tabular}

TABLE 3 . Genus-4 curves over small finite fields with many points.

Note that for $q=79$ and $q=97$ the exponent on $z$ is 3 , not 2 .

Remark 6.3. For our particular cases $(q, N)=(11,34)$ and $(q, N)=(61,120)$, there is also a more computational approach to showing that there is no genus-4 curve $C$ over $\mathbf{F}_{q}$ with $N$ points. As we have seen, we may assume that there is a fifth root of unity in the center of the endomorphism ring of the Jacobian of $C$, and it follows that $C$ has an automorphism of order 5 . Since the finite fields we are concerned with contain the fifth roots of unity, this shows that $C$ is a degree- 5 Kummer extension of another curve, and by using the Riemann-Hurwitz formula we see that this second curve must be the projective line. It is not hard to enumerate such Kummer covers; doing so, we find no curves with $N$ points.

\section{Lower bounds from explicit examples}

In this section we prove the new lower bounds for $N_{q}(4)$ given in Table 1 by providing examples of genus- 4 curves with many points. Each line of Table 3 gives a prime power $q$, an integer $N$, and the equations for a genus- 4 curve over $\mathbf{F}_{q}$ having $N$ points. 
Almost all of the examples in Table 3 were obtained by running our program double_cover_given_trace. The exceptions are the example for $q=47$, which was found by running double_cover_genus_4 on a carefully chosen genus-2 curve, and the examples for $q=79$ and $q=97$, which were found during a search of degree-3 Kummer covers of elliptic curves. While searching for such Kummer covers, we also found a particularly nice example for $q=67$ : the curve $y^{6}=x^{3}+x-6$ attains $N_{67}(4)$.

The function check_examples in the file Genus4.magma verifies that all of these examples do have the number of points claimed.

\section{References}

[1] Z. I. Borevič and D. K. Faddeev, Representations of orders with cyclic index, Trudy Mat. Inst. Steklov 80 (1965), 51-65; English transl. in Proceedings of the Steklov Institute of Mathematics. No. 80 (1965): Algebraic number theory and representations (1968).

[2] Wieb Bosma, John Cannon, and Catherine Playoust, The Magma algebra system. I. The user language, J. Symbolic Comput. 24 (1997), no. 3-4, 235-265. Computational algebra and number theory (London, 1993). DOI: 10.1006/jsco.1996.0125

[3] Pierre Deligne, Variétés abéliennes ordinaires sur un corps fini, Invent. Math. 8 (1969), 238-243. DOI: 10.1007/BF01406076

[4] Max Deuring, Die Typen der Multiplikatorenringe elliptischer Funktionenkörper, Abh. Math. Sem. Hansischen Univ. 14 (1941), 197-272.

[5] Robert M. Guralnick and Everett W. Howe, Characteristic polynomials of automorphisms of hyperelliptic curves, Arithmetic, geometry, cryptography and coding theory, Contemp. Math., vol. 487, Amer. Math. Soc., Providence, RI, 2009, pp. 101-111.

[6] H. Hasse, Zur Theorie der abstrakten elliptischen Funktionkörper. I: Die Struktur der Gruppe der Divisorenklassen endlicher Ordnung, J. Reine Angew. Math. 175 (1936), 5562; II: Automorphismen und Meromorphismen. Das Additionstheorem, J. Reine Angew. Math. 175 (1936), 69-88; III: Die Struktur des Meromorphismenringes. Die Riemannsche Vermutung, J. Reine Angew. Math. 175 (1936), 193-208. DOI: 10.1515/crll.1936.175.55, 10.1515/crll.1936.175.69, 10.1515/crll.1936.175.193

[7] Everett W. Howe, Principally polarized ordinary abelian varieties over finite fields, Trans. Amer. Math. Soc. 347 (1995), 2361-2401. DOI: 10.2307/2154828

[8] E. W. Howe and K. E. Lauter, Improved upper bounds for the number of points on curves over finite fields, Ann. Inst. Fourier (Grenoble) 53 (2003), no. 6, 1677-1737. DOI: 10.5802/aif.1990. Corrigendum, 57 (2007), no. 3, 1019-1021. DOI: 10.5802/aif.2284

[9] Everett W. Howe and Kristin E. Lauter, New methods for bounding the number of points on curves over finite fields, Geometry and Arithmetic (Schiermonnikoog, 2010) (C. Faber, G. Farkas, and R. de Jong, eds.), European Mathematical Society. To appear. Preprint version: arXiv:1202.6308v2 [math.NT]

[10] Everett W. Howe, Enric Nart, and Christophe Ritzenthaler, Jacobians in isogeny classes of abelian surfaces over finite fields, Ann. Inst. Fourier (Grenoble) 59 (2009), no. 1, 239-289. DOI: 10.5802/aif.2430

[11] Yasutaka Ihara, Some remarks on the number of rational points of algebraic curves over finite fields, J. Fac. Sci. Univ. Tokyo Sect. IA Math. 28 (1981), no. 3, 721-724 (1982). http://hdl.handle.net/2261/6319

[12] Gilles Lachaud, Christophe Ritzenthaler, and Alexey Zykin, Jacobians among abelian threefolds: a formula of Klein and a question of Serre, Math. Res. Lett. 17 (2010), no. 2, 323-333. http://www.mrlonline.org/mrl/2010-017-002/2010-017-002-011.html

[13] Kristin Lauter, The maximum or minimum number of rational points on genus three curves over finite fields, Compositio Math. 134 (2002), no. 1, 87-111. With an appendix by JeanPierre Serre. DOI:10.1023/A:1020246226326.

[14] Yu. I. Manin, What is the maximum number of points on a curve over $\mathbf{F}_{2}$ ?, J. Fac. Sci. Univ. Tokyo Sect. IA Math. 28 (1981), no. 3, 715-720 (1982). http://hdl.handle.net/2261/6318.

[15] J. S. Milne, Jacobian varieties, Arithmetic geometry (Storrs, Conn., 1984), Springer, New York, 1986, pp. 167-212. http://jmilne.org/math/articles/index.html\#1986c 
[16] Alexander Schiemann, Classification of Hermitian forms with the neighbour method, J. Symbolic Comput. 26 (1998), no. 4, 487-508. DOI: 10.1006/jsco.1998.0225, online tables available at http://www.math.uni-sb.de/ag/schulze/Hermitian-lattices/

[17] Jean-Pierre Serre, Sur le nombre des points rationnels d'une courbe algébrique sur un corps fini, C. R. Acad. Sci. Paris Sér. I Math. 296 (1983), no. 9, 397-402. = CEuvres [128]. http://gallica.bnf.fr/ark:/12148/bpt6k55351747/f35

[18] Nombres de points des courbes algébriques sur $\mathbf{F}_{q}$, Seminar on number theory, 19821983 (Talence, 1982/1983), Univ. Bordeaux I, Talence, 1983, pp. Exp. No. 22, 8. = (Euvres [129].

[19] Résumé des cours de 1983-1984, Ann. Collège France (1984), 79-83. = CEuvres [132].

[20] S. G. Vlèduts and V. G. Drinfel'd, The number of points of an algebraic curve, Funktsional. Anal. i Prilozhen. 17 (1983), no. 1, 68-69; English transl., Functional Anal. Appl. 17 (1983), no. $1,53-54$. DOI: $10.1007 /$ BF01083182

[21] William C. Waterhouse, Abelian varieties over finite fields, Ann. Sci. École Norm. Sup. (4) 2 (1969), 521-560. http://www.numdam.org/item?id=ASENS_1969_4_2_4_521_0

[22] André Weil, Sur les fonctions algébriques à corps de constantes fini, C. R. Acad. Sci. Paris 210 (1940), 592-594. http://gallica.bnf.fr/ark:/12148/bpt6k31623/f592

[23] _ On the Riemann hypothesis in function-fields, Proc. Nat. Acad. Sci. U. S. A. 27 (1941), 345-347. http://www.pnas.org/content/27/7/345.short

[24] Sur les courbes algébriques et les variétés qui s'en déduisent, Actualités Sci. Ind., no. 1041 = Publ. Inst. Math. Univ. Strasbourg 7 (1945), Hermann et Cie., Paris, 1948.

[25] — Variétés abéliennes et courbes algébriques, Actualités Sci. Ind., no. $1064=$ Publ. Inst. Math. Univ. Strasbourg 8 (1946), Hermann \& Cie., Paris, 1948.

Center for Communications Research, 4320 Westerra Court, San Diego, CA 921211967, USA.

E-mail address: however@alumni.caltech.edu

$U R L:$ http://www.alumni.caltech.edu/ however/ 